\title{
EFEITOS DO EXERCÍCIO FÍSICO NO TRATAMENTO DA OBESIDADE NA IDADE ADULTA
}

\author{
EFFECTS OF PHYSICAL EXERCISE ON THE TREATMENT OF OBESITY IN ADULT AGE
}

\section{Karolynne Rocha Nascimento ${ }^{1}$ \\ Ayer Barsanulfo Franco ${ }^{2}$}

RESUMO: O respectivo estudo possui por finalidade maior, oferecer importantes informações acerca de apontar quais são os principais efeitos positivos que os Exercícios Físicos podem ofertar, aos sujeitos adultos. Assim, faz-se importante destacar a relevância que há na prática regular, sistematizada e orientada de EF no que tange a prevenção e/ou tratamento de diversas doenças, ocasionadas pelo fator Obesidade. Portanto, o objetivo geral desse estudo vai de encontro a realizar um maior entendimento frente ao diálogo bibliográfico com importantes autores teórico-científicos acerca de identificar quais são os principais efeitos do exercício físico no tratamento da obesidade, para os sujeitos adultos. O procedimento metodológico desse trabalho encontra-se disposto em formato de Revisão de Literatura, sobe um diálogo exploratório-bibliográfico, de cunho qualitativo. Portanto, conclui-se que, os hábitos saudáveis, uma alimentação balanceada, somados a prática de exercícios regulares e prescritos por um profissional de Educação Física, são condutas de suma importância para prevenir os sintomas relacionados á Obesidade, na população adulta, resultando também assim, em significativas melhoras, na qualidade de vida.

Palavras-Chave: Exercícios Físicos. Obesidade. Sujeitos adultos. Doenças. Qualidade de vida.

ABSTRACT: The respective study has the main purpose of offering important information about pointing out the main positive effects that Physical Exercises can offer to adult subjects. Thus, it is important to highlight the relevance of the regular, systematized and guided practice of $\mathrm{PE}$ with regard to the prevention and/or treatment of various diseases caused by the Obesity factor. Therefore, the general objective of this study is to achieve a greater understanding in front of the bibliographic dialogue with important theoretical-scientific authors about Identifying what are the main effects of physical exercise in the treatment of obesity, for adult subjects. The methodological procedure of this work is arranged in the Literature Review format, under an exploratorybibliographic dialogue, of a qualitative nature. Therefore, it is concluded that healthy habits, a balanced diet, added to the practice of regular exercises and prescribed by a Physical Education professional, are extremely important conducts to prevent symptoms

\footnotetext{
${ }^{\mathrm{I}}$ Acadêmica do $7^{\circ}$ período do Curso de Educação Física da Faculdade UniBrás - Unidade Rio Verde. Email: karolynnerochanascimento@gmail.com.

${ }^{2}$ Tutor na UFSM-RS. E-mail: ayerifgoaino@gmail.com.
} 
related to obesity in the adult population, also resulting in this in significant improvements, in the quality of life.

Keywords: Physical Exercises. Obesity. Adult subjects. Illnesses. Quality of life.

\section{INTRODUÇÃO}

Indivíduos com uma rotina diária cansativa, que fazem grandes esforços para lidar com trabalho, estudo e atividades domésticas, em suma, colocam os Exercícios Físicos como quesito final da lista de interesses, prioridade e/ou preocupações, porém far-se-á de extrema necessidade repensar suas prioridades.

Diante dessas premissas, a importância do EF para a saúde e qualidade de vida na fase adulta, torna-se enorme e isso pode ser visto por meio dos benefícios que a prática regular de exercícios físicos propicia a seus praticantes. Um dos benefícios é o aumento significativo da disposição e o melhor não é preciso levar o corpo ao extremo, praticando ao menos um alongamento, caminhada ou até mesmo uma corrida leve durante 30 minutos por dia já é o bastante para se perceber os resultados gradualmente previstos.

Frente a essas considerações, o respectivo estudo possui por intenção maior, o de contribuir com significativas informações sobre os efeitos do Exercício Físico no tratamento da obesidade na idade adulta, destacando a importância que há nessas atividades se prescritas de forma orientada, por um profissional da saúde, em especial e objeto desse estudo, do Educador Físico.

Nesta perspectiva, o problema deste estudo caminha em buscar responder a seguinte questão: "De que forma os exercícios físicos podem contribuir para a vida dos sujeitos obesos na fase adulta?”

Uma das hipóteses para a resposta desse questionamento está ligada nas questões que o exercício físico regular está associado com diversos benefícios à saúde, seja de imediatos e/ou em longo prazo tais como: controle de peso, melhora da capacidade cardiorrespiratória, bem estar psicossocial, dentre outros.

Inúmeras pesquisas afirmam que a inatividade física não afeta apenas em relação à qualidade de vida, mas também tem se transformado em um fator de risco para diversas 
doenças, e isso associado à alimentação inadequado tem aumentado grandemente o potencial de perigo que estes aspectos contêm.

Dessa forma, o tema abordado se justifica em acreditar ser ele de suma importância para toda a população, sendo um tema de suma relevância para a saúde pública, sendo que isto afeta áreas como psicologia, educação física, medicina, dentre outras.

Com a incessante busca pelo aumento da qualidade de vida cada vez mais se tem realizado pesquisas referentes ao assunto supracitado nesse trabalho, de forma que atualmente relata-se que este conceito é a integração de diferentes aspectos referentes ao corpo humano. Para se obter uma qualidade de vida mínima necessita-se que exista um equilíbrio entre corpo e mente, sendo que no aspecto mental tem-se o equilíbrio das emoções, e referente ao corpo leva-se em consideração que a pessoa deve manter hábitos saudáveis, como uma alimentação saudável e balanceada além de realizar exercícios físicos com frequência.

$\mathrm{Na}$ atualidade, o sedentarismo, alimentação inadequada, a inatividade física, a genética, o tabagismo, excesso de peso, dentre outros, são fatores de risco cardiovascular, e que dessa forma, estão intimamente associados à inúmeras doenças crônicas e cardiovasculares.

Portanto, esse estudo procurou ter como objetivo geral, realizar um maior entendimento frente ao diálogo bibliográfico com importantes autores teórico-científicos acerca identificar quais são os principais efeitos do exercício físico no tratamento da obesidade, para os sujeitos adultos.

Já os objetivos específicos são: - Descrever o que é obesidade; - Relatar o que é exercício físico; - Apontar a influência do exercício físico na saúde humana; - Contribuir com esse tema em prol da sociedade como um todo, para outras pesquisas.

Para a estrutura, sustentação e embasamento desta pesquisa, utilizou-se um estudo exploratório/bibliográfico, de natureza qualitativa onde, através da análise de documentos, realiza-se novas leituras e interpretação desses estudos sendo extraída dos estudos de fontes secundárias, tratando-se assim de um levantamento de bibliografias publicadas em livros, jornais, revistas, documentos eletrônicos, dentre outras fontes que contribuíram para a estrutura e fundamentação teórico-científica deste trabalho. 
Desta forma, a metodologia executada nesse estudo, encontra-se caracterizada por análises de fontes disponíveis, apresentadas em formato de artigos científicos, teses ou dissertações defendidas por autores com considerável experiência no assunto aqui abordado, onde estas metodologias propõem ao pesquisador aproximar-se o mais possível do fenômeno a ser pesquisado, que neste trabalho está condicionado a refletir sobre a importância da prática regular de exercício físico para diminuir nos indivíduos obesos, problemas de saúde, prevenindo doenças e melhorando significativamente, sua qualidade de vida.

\section{OBESIDADE}

A obesidade na atualidade é vista como a doença epidêmica do século, haja vista o grande contingente de pessoas que se encontram acima do peso em diversos países do mundo, especialmente os mais industrializados (ENES \& SLATER, 2010).

A obesidade é uma doença complexa com consequências sociais e psicológicas graves, que afeta todas as idades e grupos sociais (COSTA et al., 2009). Assim, o excesso de peso e a obesidade podem ser considerados um dos mais sérios problemas enfrentados pelos países nos últimos tempos. A obesidade atualmente pode ser considerada como um problema de saúde pública que vem crescendo consideravelmente.

A obesidade é uma doença, catalogada como tal no Código Internacional de Doenças (CID). O excesso de gordura corporal acumulada pode atingir graus capazes de afetar a saúde. Além disso, destaca-se com enorme prevalência nos países desenvolvidos, atingindo homens e mulheres de todas as etnias e idades, reduzindo a qualidade de vida e apresentando elevadas taxas de morbidade e mortalidade (OMS, 2006).

Segundo Enes \& Slater (2010) a obesidade pode ser definida, de uma maneira simplificada, como o acúmulo excessivo de gordura corporal, sob a forma de tecido adiposo, sendo consequência de balanço energético positivo, capaz de acarretar prejuízo à saúde dos indivíduos. Sabe-se ainda que a etiologia da obesidade é multifatorial, estando envolvidos em sua gênese tanto aspectos ambientais como genéticos.

O acesso rápido e fácil a alimentos "não saudáveis" e a pouca prática de atividades físicas devido à modernização, estão levando as pessoas a habituarem-se a um estilo de 
vida sedentário.

De acordo com dados do Instituto Brasileiro de Geografia e Estatística (2010) mostram que um em cada dez adultos é considerado obeso e há tendência em aumentar esta proporção já que muitas doenças deste século, as chamadas doenças da era moderna, estão diretamente relacionadas ao excesso de gordura corporal. Como exemplo pode-se citar: doenças cardiovasculares, renais, digestivas, diabetes, problemas hepáticos e ortopédicos. A incidência dessas doenças é cerca de duas vezes maior entre os homens obesos e quatro vezes maior entre as mulheres obesas, quando comparados à população não obesa (IBGE, 2010).

Santos et al., (2006) afirmam que as causas que contribuem para o desenvolvimento da obesidade nos sujeitos, estão também o ganho excessivo e progressivo de peso e uma dieta rica em carboidratos refinados, gorduras saturadas e pobre em fibras alimentares, promovendo dessa forma, a aterosclerose, aumentando o risco de doenças cardiovasculares.

Ainda Santos et al., (2006, p.4) corroboram em afirmar que:

O excesso de gordura corporal, de modo particular a obesidade abdominal, o sedentarismo e a predisposição genética podem promover a resistência à insulina, que está intimamente relacionada à síndrome metabólica, porém os mecanismos para tal associação não estão bem esclarecidos, todavia em resposta à resistência tecidual, a secreção de insulina é aumentada, resultando em hiperinsulinemia ${ }^{4}$ (SANTOS et al., 2006, p. 4).

Portanto, o elevado índice de gordura corporal leva ao acúmulo de lipídeos nos tecidos, notadamente no tecido adiposo, músculos fígado e células pancreáticas o que parece compelir ações bioquímicas, ocasionando sérias doenças (MARCON e NEUMAN, 2011).

Rocha et al., (2009) muito contribuem em definir Exercício Físico como toda e qualquer Atividade Física planejada, estruturada e repetitiva que tem por objetivo maior, a melhoria e manutenção de um ou mais componentes da aptidão física, como podemos citar como exemplos uma caminhada de uma hora sem interrupção e de caráter contínuo, atividades aquáticas e caminhadas sistematizadas, dentre tantos outros que se forem desenvolvidos com sequência de movimentos corporais, realizados assim pelos músculos corporais, para alcançar-se um objetivo, estando ligados seja pela saúde, ou até mesmo pela estética, e que por conseguinte, resultem ao gasto de energia em valores superiores ao gasto 
em repouso, são considerados como exercícios físicos.

Porém, Vigitel (2013) afirma que, mesmo os exercícios físicos apresentando elementos distintos e comunhão com as atividades físicas, eles são diferentes, e dessa forma, o Exercício físico é considerado uma subcategoria da Atividade Física, levando-se em consideração que os Exercícios Físicos são toda e qualquer Atividade Física planejada, sistematizada, estruturada e repetitiva, cuja finalidade maior culmina em melhorar e promover a manutenção de um ou mais elementos que compõem a atividade física.

\section{A INFLUÊNCIA DO EXERCÍCIO FÍSICO NA SAÚDE HUMANA}

Para Medina (2014) os Exercícios Físicos trazem diferentes benefícios para a saúde, evitando assim a inúmeras doenças, bem como auxiliam no controle do peso corporal, na perda de gordura, diminuição da pressão arterial, redução do estresse, além de prevenir doenças como as cardiovasculares e o diabetes, desde que seja realizado regularmente.

Pontua Marcon (20II) que inúmeras pesquisas epidemiológicas e clínicas têm demonstrado e comprovado nas duas últimas décadas que, a prática regular de Exercícios Físicos é uma importante ferramenta, para a prevenção e tratamento dos fatores de risco que podem acarretar doenças cardiovasculares, bem como as DCNT (Doenças Crônicas Não Transmissíveis), onde podemos citar como exemplo de DCNT, o Diabetes tipo II.

Ainda Ciolac; Guimarães (2004, p. 3) fomentam que:

Os Exercícios Físicos devem ter prescrição orientada e executada, de forma a determinar um gasto energético bem maior do corpo comparado aos níveis de repouso, indicando novos ajustes fisiológicos e novos estímulos, o que derivará em alterações orgânicas benéficas, aprimorando as condições físicas gerais. Podendo proporcionar melhora psicossocial na qualidade de vida dos indivíduos (CIOLAC; GUIMARÃES, 2004, p.3).

Segundo Vasconcelos et al., (2013) aos sujeitos obesos e adultos, faz-se extremamente necessários ser descrito um programa de exercícios físicos cuja finalidade maior seja a de melhorar sua qualidade de vida, aprimorando também sua aptidão física, frente ao tratamento e a prevenção de inúmeras doenças relacionadas com a obesidade, contando assim com a intervenção de um profissional, que deverá em suas orientações profissionais incluir três elementos de suma importância, sendo eles o treinamento aeróbico, treinamento de força e treinamento de flexibilidade. 


\section{EFEITOS DO EXERCÍCIO FÍSICO NO TRATAMENTO DA OBESIDADE PARA OS SUJEITOS ADULTOS}

Para ABESO (2010) "O tratamento da obesidade fundamenta-se nas intervenções para modificação do estilo de vida, na orientação dietoterápica, no aumento da atividade física e em mudanças comportamentais”.

Segundo Pereira et al., (2012) a OMS (Organização Mundial de Saúde) diante de inúmeras pesquisas e estudos comprovou que a prática contínua, regular e diária de Exercício Físico, estando em conformidade com uma alimentação balanceada, poderá provocar uma expressiva redução da circunferência abdominal, onde dessa forma, a gordura visceral melhora de maneira muito significativa a sensibilidade à insulina, diminuindo os níveis plasmáticos de Glicose, auxiliando na prevenção e retardando o aparecimento do Diabetes tipo 2.

Marcon (20II) ressalva que com o crescente aumento da obesidade e do sobrepeso, onde muitas vezes estão relacionados aos novos e hábitos da agitação da vida moderna, vêm aumentando os números de indivíduos obesos, onde dessa forma, torna-se de suma importância a prática regular de EF, buscando-se assim, a melhora na saúde e, consequentemente, na qualidade de vida.

Frente a essas reflexões supracitadas acima, torna-se fundamental o papel do Profissional de Educação Física, no processo de prevenção e controle da Síndrome Metabólica, evitando complicações como o agravamento de doenças cardiovasculares e/ou crônicas, onde será prescrito com vasto conhecimento, um programa de Exercícios tanto aeróbicos, quanto resistidos, que muito contribuíram para que os sujeitos tenham uma vida mais saudável (VIGITEL, 2013).

Para Medina (2014) a prática regular de EF, contando com uma duração mínima de 30 minutos, de preferência que sejam diários, distribuídos em exercícios aeróbicos e anaeróbicos, são condutas de extrema importância para o equilíbrio energético, diminuindo assim tanto o risco relacionado aos fatores de risco, ligados a obesidade, reduzindo a pressão arterial, elevando o HDL-colesterol e auxiliando no controle glicêmico dos sujeitos, bem como melhorando a qualidade de vida de cada um deles. 


\section{RESULTADOS ESPERADOS}

Sobre a obesidade, como resultado da alimentação inadequada, do sedentarismo, da inatividade física, da genética, dentre outros, faz-se de extrema importância que os indivíduos compreendam sobre a importância de eliminar peso e praticar algum tipo de Exercício Físico Regular, sendo eles os EF, caracterizados como as melhores maneiras de prevenir e tratar a Síndrome Metabólica, onde detectar o problema poderá reduzir o aparecimento de futuras doenças cardiovasculares e/ou crônicas, onde também é de suma relevância que haja mudanças no estilo de vida dos sujeitos, para que seja evitado significativamente o desencadeamento de inúmeras complicações que estão em suma, relacionadas a obesidade e que no mundo todo acarreta, milhares de óbitos

Assim, buscando relacionar os exercícios físicos no favorecimento da melhoria do quadro de pacientes obesos, relacionando-se assim as referências bibliográficas, que vão contextualizar, oferecendo base teórico-científica ao tema supracitado neste trabalho, onde em outras possíveis oportunidades, este estudo servirá para outras pesquisas.

Assim, pode-se observar que a prática regular de exercícios pode ajudar a prevenir inúmeras doenças relacionadas à obesidade, como pode-se citar a Síndrome Metabólica, Diabetes Mellitus tipo II, Doenças Cardiovasculares, dentre outras.

Diversos estudos apontam que o exercício físico regular e orientado trás efeitos positivos para a população adulta e obesa, onde os EF, sob orientação de um profissional da saúde, de modo especial e objeto desse estudo, aos profissionais da Educação Física, surti efeitos benéficos e favoráveis, na redução de peso. Outro aspecto importante da prática de EF culmina na melhora da qualidade de vida desses sujeitos obesos.

\section{CONCLUSÕES}

A obesidade está relacionada ao conjunto de fatores de risco, que em suma, ao manifestar-se nos indivíduos, poderão aumentar as chances deles desenvolverem algum tipo de doenças cardiovasculares como por exemplo o AVC (Acidente Vascular Cerebral) e/ou doenças crônicas, como o Diabetes tipo II, dentre tantas outras graves enfermidades que podem levar ao óbito.

Assim, após a realização desse estudo pode-se concluir o quão far-se-á de suma 
importância que os sujeitos adultos, optem por hábitos saudáveis, aos quais compõem uma alimentação balanceada e adequada, o não uso de substâncias que contenham tabaco e álcool, a perda de peso, o rompimento com o sedentarismo, e a prática regular de Exercícios Físicos, são importantes condutas básicas que os indivíduos devem adotar, para ter uma vida mais saudável e com qualidade.

Dessa forma, o papel dos Exercícios Físicos são ferramentas de extrema importância, onde recomenda-se que pelo menos trinta minutos de atividades aeróbicas regulares, diárias e contínuas, mesmo que esses EF prescritos de preferência por um profissional do campo da Educação Física, a princípio não poderá promover uma significativa perda de peso, porém haverá a redução do tecido adiposo visceral, melhorando assim a homeostase da glicose, bem como o transporte de glicose e a ação da insulina na musculatura em exercício, e assim, outro ponto positivo na prática regular de Exercícios Físicos, culmina na melhoria do perfil lipídico, aumentando dessa forma, os níveis de HDL-colesterol, bem como a diminuição dos níveis de triglicérides, auxiliando aos sujeitos adultos, uma melhoria significativa em sua qualidade de vida.

\section{REFERÊNCIAS BIBILOGRÁFICAS}

ABESO - ASSOCIAÇÃO BRASILEIRA PARA O ESTUDO DA OBESIDADE E SÍNDROME METABÓLICA-ABESO. Atualização das Diretrizes para o Tratamento Farmacológico da Obesidade e do Sobrepeso. Edição Especial, Outubro, 2010.

CIOLAC, Emmanuel Gomes. GUIMARÂES, Veiga Guimarães. Exercício físico e síndrome metabólica. Ver. Bras Med Esporte _ Vol. ı, № 4-Jul/Ago, 2004. Disponível em: https://www.scielo.br/pdf/rbme/vion4/22048. Acesso em: I6 de Out. de 2021.

COSTA, A. P. P. L. Obesidade feminina: um estudo de sua relação com a sexualidade. Brasília: Universidade Católica de Brasília, 2006. Dissertação de Mestrado em Psicologia.

ENES, C. C.; SLATER, B. Obesidade na adolescência e seus principais fatores determinantes. Revista Brasileira Epidemiológica. São Paulo, v.13, n.I, p. I63-17I, 
março/2oıo. Disponível em: http://www.scielo.br/scielo.php?. Acesso em: is de Out. de 2021.

GIL, A. C. Como elaborar projetos de pesquisa. São Paulo: Atlas, 2010.

IBGE - Instituto Brasileiro De Geografia E Estatística. Pesquisa de orçamentos familiares: POF 2002-2003. Disponível em: http://www.ibge.gov.br/. Acesso em: 30 de Set. 202I.

MARCON, E. R; GUS, I.; NEUMANN, C.R. Impacto de um programa mínimo de exercícios físicos supervisionados no risco cardiometabólico de pacientes com obesidade mórbida. ArqBrasEndocrinolMetab., vol.5, n.55, pp. 331-338, 2011.

MARCONI, M. A.; LAKATOS, E. M. Metodologia do trabalho científico: procedimentos básicos, pesquisa bibliografia, projeto e relatório, publicações e trabalhos científicos. 6.ed. São Paulo: Atlas, 2017.

MEDINA, Fábio. Você sabe a diferença entre atividade física e exercício físico? Disponível em: http://www.fabiomedina.com.br/fabio-medina-assessoria-esportiva/ı7ovoce-sabe-a-diferen $\% \mathrm{C}_{3} \% \mathrm{~A}$ 7a-entre-atividade-f\% $\mathrm{C}_{3} \% \mathrm{ADsica}-\mathrm{e}-\mathrm{exerc} \% \mathrm{C}_{3} \% \mathrm{ADcio}-$ f\% $\mathrm{C}_{3} \% \mathrm{AD}$ sico. Acesso em: 03 de Out. de 2021.

OMS - ORGANIZAÇÃO MUNDIAL DA SAÚDE. Obesidade: Prevenindo e Controlando a Epidemia Global. São Paulo: Roca, 2006.

PEREIRA, F. M et al., Melhora do Perfil Lipídico Através do Exercício Físico. Rev. Bras. Med. Sport, Blumenau, Nov/Dez, pp. I-5, 2012.

SANTOS C. R. B et al., Fatores dietéticos na prevenção e tratamento de comorbidades associadas à síndrome metabólica. Revista de Nutrição, Campinas, I9(3):389-40I, maio/jun., 2006. Disponível em: https://www.scielo.br/pdf/rn/vign3/30144.pdf. Acesso em: 02 de Out. de 202I. 
VASCONCELOS, F. V. A.. et al., Exercício físico e síndrome metabólica. Revista HUPE, Rio de Janeiro, 2013;12(4):78-88 doi:10.12957/rhupe.2013.8715. Disponível em: file://C:/Users/Victor/Downloads/8715-42160-I-PB.pdf. Acesso em: or de Out. de 2021.

VIGITEL, Brasil. Vigilância de Fatores de Risco e Proteção para Doenças Crônicas por Inquérito Telefônico. Ministério da Saúde, Brasília, 2013. 